\title{
An Approach for Restoring Occluded Images for Face-Recognition
}

\author{
Sandesh V Khamele ${ }^{1}$, Shyamal G Mundada ${ }^{2}$ \\ M.tech scholar, Department of Computer Science, SRCOEM, Nagpur, India ${ }^{1}$ \\ Asst. Professor, Department of Computer Science, SRCOEM, Nagpur, India ${ }^{2}$
}

\begin{abstract}
The Systems that rely on Face Recognition (FR) biometric have acquired great importance ever since terrorist threats injected weakness among the implemented security systems. Rest biometrics as iris or Fingerprints recognition is not trustworthy in such situations whereas FR is considered as a better compromise. In Image processing, Occlusion refers to facade of the face image which can be due to hair, moustache, sunglasses, or wrapping of facial image by scarf or other accessories. Efforts on FR appears in controlled environment have been in the picture for past several years; however identification under uncontrolled condition like partial occlusion is typically quite a matter of concern. Based on review of literature and its analysis so far, a classification made in this paper to solve the challenges in recognition of face in the presence of partial occlusion. The methods used are INPAINTING based methods that make use of Exemplar-based Inpainting, Feature-Extraction, and Fast Weighted-Principal component analysis (FWPCA),etc.

The presented approach in this paper describes the removal of Occlusion from images or restore the occluded part of image using Exemplar-based Image Inpainting technique, feature extraction and FW-PCA(Restoration) combinations.
\end{abstract}

Keywords: Occlusion; Image Inpainting; Exemplar based inpainting technique, Face-detection, Feature extraction, Image Restoration, FW-PCA.

\section{INTRODUCTION}

Though tracking and recognizing face objects is a routine task for humans and building such a computerised system OCCLUSION is defined as an Obstacle or any are still an active research. Despite several proposed face unnecessary-object in image disturbing the matching sometimes called recognizing process. Occlusion in an image refers to hindrance in the view of an object. recognition schemes, image based approaches are possibly Occlusion observed can normally be natural as well as the most promising ones. However, the images patterns of synthetic. Natural occlusion indicates to obstruction of face objects can dramatically change due to occlusion views between the two image objects without any type, lighting and viewing variations. The major portion intension while synthetic occlusion refers to artificial due to which conflict occurs while Face recognition, is blockade of intentionally covering the image's view with a facial occlusion. The research for removal of such facial white/black solid rectangular block. Partial occlusion has occlusion using restoration or reconstruction of specific been observed in many areas of image processing. It is area is currently an active field. Here, system is proposed found in iris recognition where the eyelashes occlude the where it is possible to remove occlusion from faces and iris; identification via ear can also be occluded by the person may be predicted.

presence of earrings. Even in real time application face

image intentionally becomes occluded via accessories such The paper is organized as follows: Section 2 comprises as

- Sunglasses, scarf, beards, hat.

- Hand on face

- Face dirt

- Face behind Fence

- Texture on Face images, etc. review of related work done; section 3 comprises Proposed work and flow of project; section 4 describes Implementation work; section 5 states Conclusion and Future work.

Digital Image Processing (DIP) is the analysis and An In jan-2014, Christine Guillemot and Olivier Le Meur manipulation of digitized image, especially in order to presented two major approaches in "IMAGE improve its quality and parameters. Almost all of the INPAINTING -Overview and recent advances". They technical fields are impacted by DIP. It is widely used in proved that system comprise of Diffusion-based Medical Field, Video processing, robot vision, Remote inpainting or Examplar-based inpainting techniques have Sensing, etc. Recently the research areas in Images major application as image disocclusion and such systems processing recently acquired a lot of attention in analysis may be discovered in order to improve results and of biometrics such as Iris, Fingerprint, Hand, Ears, Face- accuracy[1].

Recognition.

Tomoki Hosoi, Sei Nagashima and Koichi Ito[2] contributed a lot in reconstruction of occluded images and 
finding most approximated matched images. They Experiments demonstrate that the proposed approach followed popular techniques of PCA and FW-PCA for significantly improve various face recognition algorithms Face Recognition and Identified FW-PCA providing in presence of complex sparse occlusions[7]. appropriate weights to sample images. Facial Occlusion is a critical problem for Face recognition. Addressing this problem, they proposed a method for restoring occluded regions in face images. The proposed method employs Fast Weighted Principal Component Analysis (FW-PCA), which computes PCA only with effective pixels. The adoption of FW-PCA makes it possible to detect and restore occluded regions in face images.

Through a set of experiments using public face databases, they demonstrated the effectiveness of the proposed method compared with the conventional methods.

Mahroosh Banday and Richa Sharma, in July-13, presented "Image Inpainting- An Inclusive Review of the Underlying Algorithm and Comparative Study of the Associated Technique " in which A comparative study to provide a comprehensive visualization of different types of image inpainting techniques were discussed[3].

Shermina .J and V. Vasudevan in Jan-12 proposed a system of an Efficient Face Recognition system based on Partial Occlusion and expression, the face they recognized by using the PCA. From the implementation result, it was proved that, proposed method recognizes the face images effectively [4].

Marcelo Bertalmio and Guillermo Sapiro, proposed a robust method of "Image Inpainting" in which they introduced a novel algorithm for digital inpainting of still images that attempts to replicate the basic techniques used by professional reconstructors.

After the user selects the regions to be restored, the algorithm automatically fills in these regions with information surrounding them.

Their experimental results stated that 'The inpainting algorithm presented has clearly motivated by and has borrowed from the intensive work on the use of $\mathrm{KNN}$ algorithm in image processing[5].

Aleix M. MartõÂnez, Member of IEEE in june-2002 described a system To make the recognition system less sensitive to the differences between the facial expression displayed on the training and the testing images, they weight the results obtained on each local area on the basis of how much of this local area is affected by the expression displayed on the current test image[6]. In dec-2012, Rui Min and Jean-Luc Dugelay presented the based Image-Impainting technique where KNN (K-Nearest system where solution to newly identified facial occlusion Neighbour) algorithm works on pixels to replaced problem viz. sparse occlusion in the context of Face occluded pixels with reference to background pixels. Just Biometrics in Video surveillance.

\section{III.PROPOSED SYSTEM MODEL}

The working model for proposed system is as follows:-

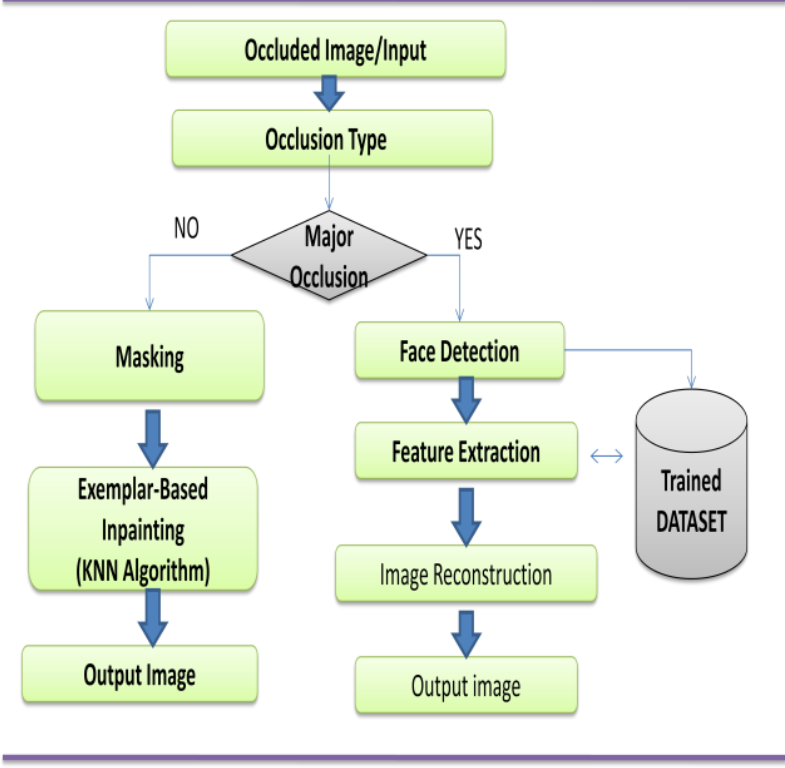

Fig 1:- Project Flow

The Process is initiated by Selecting the input image which is found occluded from the system simply browsing the required path.

As the selected image get labelled, Our approach is to visualize the image and classify on the basis to occlusion and classify in the category of Minor occlusion or major occlusion.

Minor Occlusion - It is an occlusion type where regular and effective pixels is get occluded by contradict pixels on texture part of image which could be impainted using their background pixels. Eg. Tattoo, beard, moustache, etc.

Major Occlusion - This category consist of occlusions which occupies some hindrance part of typical recognizable feature of face like eye, nose, mouth, etc behind, and which is not possible to impaint for recovery, such occlusions are called Major occlusions. Eg. Goggle, Scarf, masks, etc.

As the input image is categorized, it would be then travelled though proper channel for manipulation.

i) If occlusion is Minor, then with the help of ImageImpainting technique it remove occluded pixels to get occlusion-free image. Here, proposal is to use Exemplar in single iteration it retrieve image which seems original and cleaned image as it had never be occluded. 
ii) In case II, consider the occlusion as major which is not coloured images of different size etc. that is given to image possible to recover with above process, then it have to be inpainting.

processed by another process of feature extraction shown Masking of Image:- It covers the region of unwanted in project flow. Major occlusion images has to be followed object either single or multiple if required from the by three process:- a) Face-Detection b) Feature Extraction original image through paint called mask image or target and c) Image Reconstruction.

which is to be inpainted.

\section{A. Face-Detection}

The input image is to be normalized first before get proceeded to further steps. Normalization leads to image detected face compare with dataset which again get trained with the help of this process on the other hand. Here, only facial part is detected and extracted from overall image excluding background area. The output image obtained is normalized and relevant to undergo further process.

\section{B. Feature Extraction}

This module takes input image obtained by Face detection which is normalized. In this, image feature get extracted in the form of vectors and represented by 66 lines of code in the .txt file. It includes feature like Color segmentation, Edges and Texture. As the feature get extracted it is then compared by features of trained dataset. The matching is contradictorily done on the basis of Differences in vectors of input image and images from dataset. The least 5 difference obtained means 5 are matching image. Here, minimum difference means maximum matching image. By obtaining matching vectors, those images are get redirected on the label.

\section{Image-Reconstruction}

From all 5 images it is possible to easily select anyone related image referring to which it can substitute the occluded part. As the Occluded pixels of input image get substituted by pixels of matching image. After the process it return the output image seems to be dis-occluded.

The input image which was occluded undergoes the process, completion of which get resultant image as occlusion free way to comply with the conference paper formatting requirements is to use this document as a template and simply type your text into it

\section{IMPLEMENTATION WORK}

\section{A. Image Impainting}

Impainting is done by sampling, and by copying or joining the calculation of the different size images. Adverse to the together patches (called exemplar) taken from the known standard approach Viola-Jones rescale the detector instead part of the image, So, the corresponding methods used are of the input image and run the detector many times known as exemplar-based techniques. Exemplar-based through the image-each time with a different size. inpainting symphonizing entire patches by learning from patches in the familiar part of the image and these methods are known to be faster than PDE pixel based approaches. Input Image:- Input image given is a photograph, natural the same number of calculations whatever the size. This image and synthetic image for dealing with, that contain detector is constructed using a so-called integral image and 
some simple rectangular features redolent of Haar D. Image Reconstruction wavelets.

The normalized input image $\mathbf{x}$ which is reconstructed using the method of FW-PCA which consist of-

It uses the package of XML file containing Thousands of positive tested samples and negative tested samples which provides the algorithm better accuracy and perfection in detection.

Using this algorithm, dataset is normalized which is called trained dataset so that input image is feasibly compared with dataset images[8].

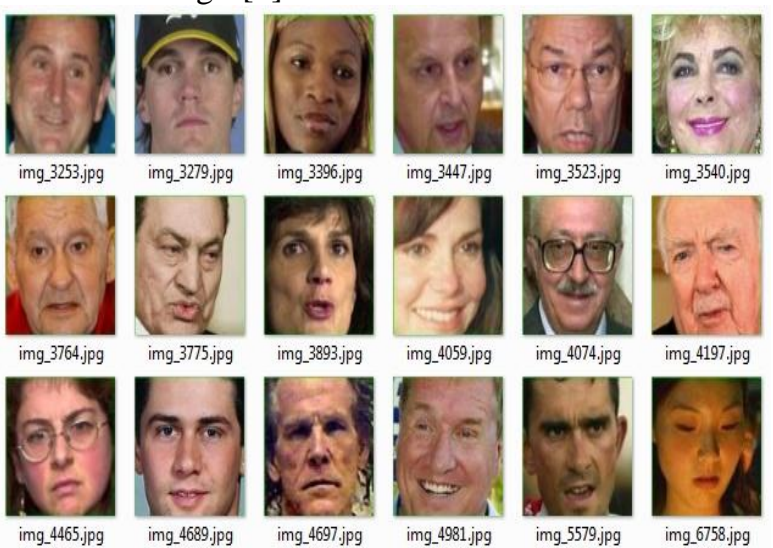

Fig 3:- Trained dataset

\section{Feature Extraction}

Feature Extraction is the key method in CBIR (Content based image retrieval). A certain number of features for each image are extracted, defining its high level content information. Then, according to the similarity of these vectors, comparisons of two specific images are estimated in numbers.

This class uses different techniques to extract features related to a single or the group of images. Two methods in this class which extracts the features:

ExtractSingleImage : which extracts the features for a single selected image and it is used to extract the features of the input image.

ExtractAll : which is used to extract the features for all images available in dataset. It takes approximate about half an hour for this method to complete and stores the result table in a text file named: "Features.txt".

FEATURE EXTRACTION PROCEDURE:-

a) The first step is the Colour Extraction which computes the feature vector consisting of 18 real numbers for each available image. Notable is that for each of these features, a normalization step is provided to fit the numbers between $0-10$.

b) Then the edge map of the given image is calculated. It is implemented in Canny Edge Detector class. It provides a binary image which consists of just zeros and ones. So, further computations will be much easier.

c) The last step is Texture Extraction. It uses cooccurrence matrix to extract texture features, and provides 48 real numbers as feature vector.

d) At last, these two vectors are concatenated to each other and a 66 length feature vector for each image is computed a) Training process :- The eigenspace $\mathbf{E}$ is computed using all the training images, The standard deviation of the reconstruction error for each pixel $\sigma=\left[\begin{array}{lll}\sigma 1 & \sigma 2 & \bullet\end{array}\right.$ $\sigma N] \mathrm{T}$ is also computed.

b) Reconstruction process :- First, the initial weight for FW-PCA is determined using the sub-samplings of the input image. $M$ pixels are extracted from the input image according to $S$ random sampling patterns. Considering the extracted pixels as the effective pixels, the reconstructed image $\mathbf{x}_{s}(s=1, \cdot \bullet \cdot, S)$ for each sampling pattern is computed by using FW-PCA i.e., Then, the reconstruction error $\mathbf{r}_{s}$ between $\mathbf{x}$ and $\hat{\mathbf{x}_{s}}$ is obtained by

$$
\mathbf{r}_{s}=\left|\mathbf{x}-\hat{\mathbf{x}}_{s}\right| \text {. }
$$

Note that the pixel values of $\mathbf{x}$ is normalized so that they have the same mean and variance

of $^{-} \mathbf{x}$ during each iteration. The pixel values of effective pixels in the final reconstructed image $\mathbf{x}$ is normalized so that they have the same mean and variance of the normalized input image $\mathbf{x}$.

c) Occluded-region restoration :- As mentioned above, image reconstruction for the whole input image is done using the eigenspace $\mathbf{E}$. The reconstructed image ${ }^{\wedge} \mathbf{x}$ may result in the smoothed image of the input image $\mathbf{x}$. Therefore, only the missing pixels of the input image $\mathbf{x}$ are replaced by the pixels of the reconstructed image ${ }^{\wedge} \mathbf{x}$. The restored image $\tilde{\mathbf{x}}$ is obtained by

$$
w_{i}=\left\{\begin{array}{ll}
1 & \text { if }\left(\left|\hat{x}_{i}-x_{i}\right|<\theta_{i}\right) \\
0 & \text { otherwise }
\end{array} \quad(i=1, \cdots, N),\right.
$$

Finally, restoration is done only on the occluded regions of the input image [2].

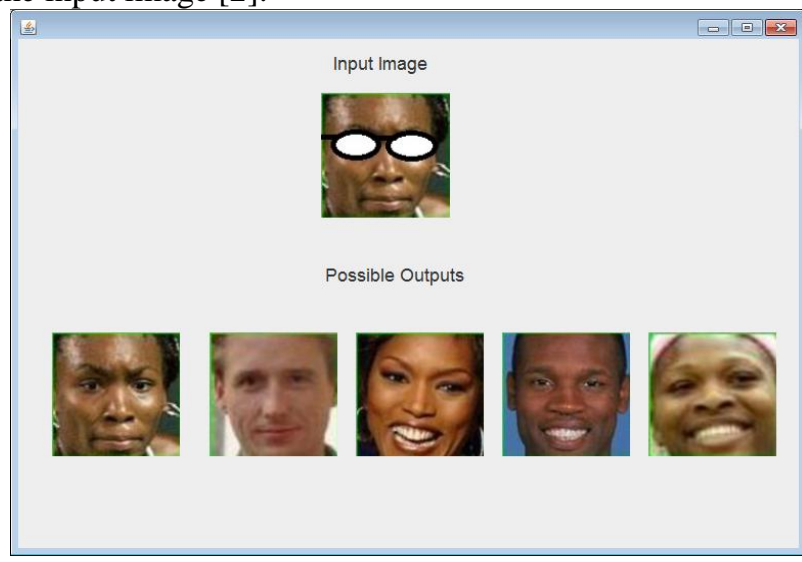

Fig 4:- Matched images

\section{Conclusion}

It is observed that Combination of KNN algorithm, Voilajones algorithm, Feature extraction and FW-PCA in a single system makes it possible to remove Facial occlusion as well as restoration of occluded images provides good results with better accuracy within seconds. Further 
processing in presented system will lead to save more processing time with the use of clustering.

Face recognition challenges would be reduced to certain extent using removal of Occlusion mechanism with quick relevant output images.

\section{REFERENCES}

[1] Christine Guillemot and Olivier Le Meur, "Image Inpainting " IEEE signal processing magazine, JANUARY 2014, 1053-5888/14.

[2] Tomoki Hosoi, Sei Nagashima and Koichi Ito, "Restoration Of Occluded Regions Using FW-PCA for Face Recognition" 978-14673-1612-5/12/\$31.00 C2012 IEEE.

[3] Mahroosh Banday and Richa Sharma," Image Inpainting- An Inclusive Review of the Underlying Algorithm and Comparative Study of the Associated Technique", International Journal of Computer Application (0975-8887) vol no 98, no 17, july 2014.

[4] S. Masnouand J. Morel, "Level-lines based disocclusion," inProc. IEEE Int. Conf. Image Processing (ICIP), Chicago, IL, Oct. 1998, vol. 3, pp. 259-263.

[5] M. Bertalmio, G. Sapiro, C. Ballester, and V. Caselles, "Image inpainting," in Proc. ACM SIGGRAPH, July 2000, pp. 417-424.

[6] Aleix M. MartõÂnez,,'Recognizing Imprecisely Localized, Partially Occluded, and Expression Variant Faces from a Single Sample per Class" IEEE TRANSACTIONS ON PATTERN ANALYSIS AND MACHINE INTELLIGENCE, VOL. 24, NO. 6, JUNE 2002.

[7] Rui Min and Jean-Luc Dugelay, "INPAINTING OF SPARSE OCCLUSION IN FACE RECOGNITION" IEEE transactions Feb. 2012

[8] Paul Viola and Michael Jones, "Robust Real Time Face Detection", Received September 10, 2001; Revised July 10, 2003; Accepted July 11, 2003.

[9] Irene Kotsia, Ioan Buciu, Ioannis Pitas, "An analysis of facial expression recognition under partial facial image occlusion" SCIENCE DIRECT Image and Vision Computing 26 (2008) 1052 $106 w i=\{7$ 